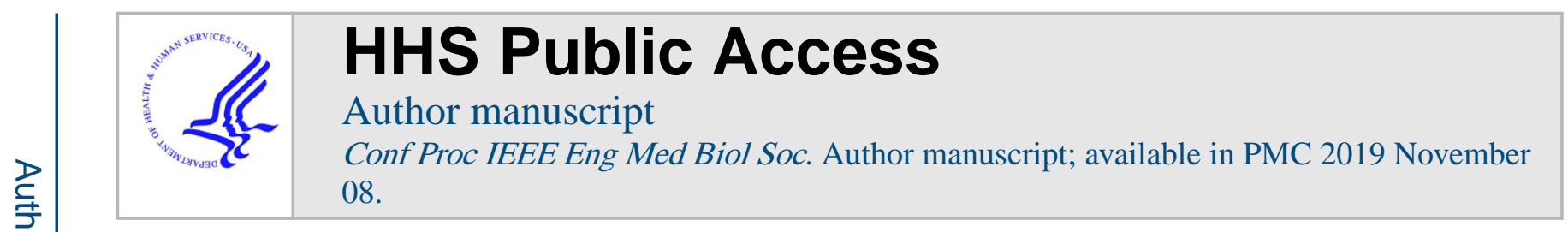

Published in final edited form as:

Conf Proc IEEE Eng Med Biol Soc. 2018 July ; 2018: 6048-6051. doi:10.1109/EMBC.2018.8513518.

\title{
Gas Embolization in a Rodent Model of Hepatocellular Carcinoma Using Acoustic Droplet Vaporization
}

\author{
Jonah S. Harmon, \\ Tulane University, New Orleans, LA 70118 USA \\ Foad Kabinejadian, \\ Tulane University, New Orleans, LA 70118 USA \\ Robinson Seda, \\ University of Michigan, Ann Arbor, MI 48109 USA \\ Mario L. Fabiilli, \\ University of Michigan, Ann Arbor, MI 48109 USA
}

Sibu P. Kuruvilla,

University of Michigan, Ann Arbor, MI 48109 USA; Stanford University, Stanford, CA 94305 USA

Joan M. Greve,

University of Michigan, Ann Arbor, MI 48109 USA

\author{
J. Brian Fowlkes, \\ University of Michigan, Ann Arbor, MI 48109 USA \\ Joseph L. Bull \\ Tulane University, New Orleans, LA 70118 USA
}

\section{Abstract}

Trans-arterial embolization is a commonly used therapy in unresectable hepatocellular carcinoma.

Current methods involve the careful placement of an intraarterial catheter and the deposition of embolizing particles. Gas embolotherapy has been proposed as an embolization method with the potential for high spatial resolution without the need for a catheter. This method involves vaporizing intravenously-administered droplets into gas bubbles using focused ultrasound - a process termed acoustic droplet vaporization. The bubbles can become lodged in the vasculature, thereby creating an embolus. Here, we initially demonstrate the feasibility of achieving significant targeted embolization with this method in the rat cremaster using intravital microscopy. The therapy was then tested in an ectopic xenograft mouse model of hepatocellular carcinoma. Gas embolotherapy was shown to maintain the tumor volume at baseline over a two-week treatment course while control groups showed significant tumor growth. These preliminary results demonstrate that gas embolotherapy could serve as an effective noninvasive method for the management of unresectable hepatocellular carcinoma.

"corresponding author: J. L. Bull (phone: 504-865-5843; jbull@ tulane.edu). 


\section{Introduction}

Intraarterial embolization is widely used to manage unresectable hepatocellular carcinoma (HCC). ${ }^{[1,3]}$ The rationale for this treatment stems primarily from the dual blood supply to the liver; it has been shown that hepatic neoplasms derive the majority of their blood supply from the hepatic artery, whereas the healthy portions of the liver receive approximately $50 \%$ of their oxygen from the portal system. ${ }^{[1,4]}$ Other factors supporting the use of intraarterial embolization include the inefficacy of systemic chemotherapy and the relatively small number of patients with resectable HCC - for example, $95 \%$ of patients in the US and $60 \%$ of patients in Asia will not qualify for resection. ${ }^{[5]}$

Current embolization methods involve the placement of an intraarterial catheter in either the hepatic artery or selectively in the tumor blood supply and the deposition of an embolizing material, such as polymer microspheres or foams. ${ }^{[1,3]}$ This is commonly combined with localized chemotherapy delivery, but with little to no added benefit over embolization alone. [6-7] Some issues with the current embolization methods arise when considering the necessity of placing an intraarterial catheter and the strict guidelines for patient qualifications; some of these, such as healthy portal venous flow and relatively healthy overall hepatic function, are necessary due to off target effects induced by large scale arterial ischemia. ${ }^{[1,3,7]}$ To circumvent these issues, an entirely noninvasive, highly spatially selective embolization method has been proposed. Gas embolotherapy utilizes focused ultrasound and a phenomenon known as acoustic droplet vaporization (ADV) to introduce gas emboli directly into the tumor vasculature. ${ }^{[8]}$

ADV involves the ultrasonically driven phase change of a perfluorocarbon droplet to a microbubble. ADV has a wide array of potential therapeutic and diagnostic uses including drug delivery, gene therapy, gas embolization, and phase change contrast agents for ultrasound imaging. ${ }^{[9-13]}$ Droplets can be produced between a size range of 1-3 $\mu \mathrm{m}$ in diameter, small enough to pass through capillaries; the diameter will increase by 5-6 times upon vaporization, allowing for embolization of small vessels. ${ }^{[14]}$ Two previous studies have investigated the use of ADV in embolotherapy specifically. ADV-induced embolization has been shown to reduce blood flow in a canine kidney, and minor embolization has been shown visually utilizing intravital microscopy in the rat cremaster. ${ }^{[15-16]}$

The current study was performed to show significant embolization and near cessation of flow in larger vessels in the rat cremaster muscle using intravital microscopy, and following confirmation of the feasibility of ADV-induced gas embolization, to demonstrate gas embolotherapy's therapeutic potential in an ectopic xenograft model of HCC.

\section{Materials and Methods}

\section{A. Droplet Preparation}

The following lipids, initially dissolved in chloroform, were combined to prepare a lipid film: 1,2-distearoyl-sn-glycero-3-phosphocholine (DSPC, $80 \mathrm{~mol} \%$, Avanti Polar Lipids, Alabaster, AL, USA), 1,2-distearoyl-sn-glycero-3-phosphoethanolamine-N[methoxy(polyethylene glycol)-2000] (ammonium salt) (DSPE-PeG, 10 mol\%, Avanti Polar 
Lipids), and 1,2-distearoyl-sn-glycero-3-hosphoethanolamine-N-[maleimide(polyethylene glycol)-2000] (ammonium salt) (DSPE-PEG-Mal, $10 \mathrm{~mol} \%$, Avanti Polar Lipids). A vacuum was used to dry the lipid mixture and remove the chloroform. A diluent consisting of phosphate buffered saline ( $80 \%$ v/v, Life Technologies, Grand Island, NY, USA), propylene glycol (10\% v/v, Sigma-Aldrich, St. Louis, MO, USA), and glycerol (10\% v/v, SigmaAldrich) was used to hydrate the lipid film; this solution was then heated to $70^{\circ} \mathrm{C}$. The final concentrations of DSPC, DSPE-PEG, and DSPE-PEG-Mal in the lipid blend were 2, 0.95, and $0.95 \mathrm{mg} / \mathrm{mL}$, respectively. Perfluoropentane (Strem Chemicals, Newburyport, MA, USA) was combined with the lipid blend in a 1:3 volumetric ratio. A sonicator (Q55, QSonica, Newton, CT, USA) was used to emulsify the phases. Repeated centrifugation was used to wash this emulsion to remove unincorporated lipids. The size distribution and concentration of the emulsion were characterized with a Coulter counter (Multisizer 4, Beckman Coulter, Brea, CA, USA). A cyclic RGD peptide (c(RGDfC), Peptides International, Louisville, KY, USA) was joined to the droplets via a maleimide linkage to the DSPE-PEG-Mal to target the integrin $a_{v} \beta_{3}$.

\section{B. Rat Preparation}

The method of rat preparation was adapted from [15]. All animal procedures were conducted with the approval and guidance of the Institutional Animal Care and Use Committee (IACUC) at the University of Michigan. Male Sprague-Dawley rats (Charles River Laboratories, Wilmington, MA, USA) weighing between 300-350 g were used. Animals were anesthetized with a mixture of isoflurane and oxygen gas. Physiological parameters were monitored and maintained within normal ranges during the experiment. A tail vein catheter was placed before using blunt dissection to expose the testicle. The cremaster was then separated from the testicle.

The rat was placed in a custom tank for intravital microscopy; the cremaster was stretched over an optical window. The tank was placed above an inverted microscope (Eclipse TE2000-S, Nikon Corporation, Tokyo, Japan) and filled with degassed saline at $37^{\circ} \mathrm{C}$.

\section{Optics and Acoustics, Cremaster}

A square wave was created by a waveform generator (33120A, Agilent, Santa Clara, CA, USA); this gated a sinusoidal wave produced by a second function generator (3314A, Hewlett-Packard, Palo Alto, CA, USA). The output was passed through a gated amplifier (60 dB, GA-2500, Ritec, Warwick, RI, USA) before reaching the transucer. An oscilloscope (Wavesurfer 44MXs-A, Teledyne LeCroy, Chestnut Ridge, NY, USA) was used to monitor the voltage waveforms applied to the transducer. A $20 \mathrm{MHz}$ sweep function generator (BK Precision 4040A, BK Precision Corporation, Yorba Linda, CA, USA), connected to the 33120A, was used to modulate the pulse repetition frequency (PRF) of the ultrasound.

A single-element 7.5-MHz transducer (A321S, Olympus Panametrics-NDT, Waltham, MA) was used. The electronic arrangement above applied 8 cycle voltage pulses, generating 2.95 MPa peak rarefactional pressure as measured in the free field using a fiber optic probe hydrophone. ${ }^{[17]}$ The PRF was varied between 100-1000 Hz. A Point Grey camera (Flea 3, FLIR Integrated Imaging Solutions, Vancouver, Canada) was used for image and video 
capture in conjunction with Point Grey FlyCap2 (2.7.3.0, FLIR Integrated Imaging Systems, Vancouver, Canada).

\section{Rat Experimental Design}

The ultrasound focus was positioned to the center of the video capture window. PRF and droplet concentrations varied by animal. The tissue was exposed to ultrasound prior to droplet injection as a negative control. An initial bolus of $0.2 \mathrm{~mL}$ of droplet solution was injected intravenously intravenously $\left(10^{8}-10^{9}\right.$ droplets $\left./ \mathrm{mL}\right)$. Video capture was initiated shortly before injection and continued until vaporization or embolization occurred. After the initial injection, $0.1 \mathrm{ml}$ injections were delivered every 5-10 minutes to attempt to vaporize further in the feeder vessel or in auxiliary vessels in the cremaster. A maximum volume of 1 $\mathrm{ml}$ was injected over the course of the experiment. The duration of the experiment, including animal preparation, was approximately 4 hours. Video and images were captured throughout the tissue. The rat was humanely euthanized according to IACUC guidelines at the end of the experiment.

\section{E. Tumor Inoculation}

HepG2, a hepatocellular carcinoma cell line, was obtained from the American Type Culture Collection (ATCC, Manassas, VA, USA). Cells were cultured in MEM supplemented with $10 \%$ fetal bovine serum, $1 \%$ antibiotic-antimycotic, $1 \%$ minimum non-essential amino acids, $1 \%$ sodium pyruvate, and $1 \mathrm{~mL}$ gentamicin; all media and additives were from Gibco Laboratories (Gaithersburg, MD, USA).

Nod Scid Gamma mice (6-8 weeks old) were maintained in accordance with both IACUC and Department of Comparative Medicine (DCM) guidelines at Tulane University. HepG2 cells in a 1:1 mixture of media to Matrigel (Matrigel ${ }^{\mathrm{TM}}$ Matrix, Corning Inc., Corning, NY, USA) were injected subcutaneously into the flank ( $150 \mu \mathrm{l}, 5$ million cells). Calipers were used to measure tumor size while monitoring growth; volume was calculated using the formula $V=1 / 2$ (length)(width) ${ }^{2[18]}$ Tumor growth was monitored for 6-8 weeks before randomly assigning a mouse to the treatment group or one of three control groups $(\mathrm{N}=5)$. The average initial tumor volume was $238.40 \pm 6.99 \mathrm{~mm}^{3}$; the initial tumor volume did not vary significantly between groups ( $\mathrm{p}>0.05$ ).

\section{F. Treatment and Imaging}

A $2.5 \mathrm{MHz}$, single element transducer (H-108, Sonic Concepts, Bothell, WA, USA) was used to insonate the tumor area. The output from the amplifier was run through an impedance matching network before being delivered to the transducer (3rd Harmonic Impedance Matching Network, Sonic Concepts, Bothell, WA, USA). The rest of the ultrasound setup matches that used in the cremaster experiments. The following ultrasound parameters were used: $5.34 \mathrm{MPa}$ peak negative pressure, 13 cycles (5.39 $\mu$ s pulse width), and $100 \mathrm{~Hz}$ PRF. A coupling cone filled with degassed water was attached to the transducer and covered with Tegaderm (3M, Maplewood, MN, USA).

A tail vein injection of droplet solution was administered prior to anesthesia $(125 \mu \mathrm{l}$, $3.5 \times 10^{6}$ droplet $\left.\mu l\right)$. Mice were anesthetized with isoflurane in oxygen gas. Photos and 
ultrasound images of the tumor were taken before and after insonation. A Zonare ultrasound system (ZS3, Mindray Medical International Limited, Shenzhen, China) with a prototype transducer operated at $25 \mathrm{MHz}$ (L25 linear array, Mindray Medical International Limited) was used for the ultrasound images. The tumor area was insonated for 2 minutes. Treatment occurred in cycles of three consecutive days followed by one day off for two weeks.

Following the treatment course, animals were humanely euthanized according to appropriate IACUC guidelines.

\section{G. Analysis}

All descriptive statistics are presented as mean \pm standard error. $\mathrm{R}$ was used for all statistical analysis. $^{[19]}$

\section{RESULTS AND DISCUSSION}

\section{A. Embolization in the Rat Cremaster Muscle}

Intravital microscopy experiments were performed using the rat cremaster muscle to visually examine the feasibility of forming gas emboli using ADV. Consistent vaporization and occlusion were both seen with each animal $(\mathrm{N}=5)$, with some qualitatively observed differences; vaporization and embolization were likely positively correlated with PRF and the concentration of the droplet solution, but further studies need to be performed to quantify this relationship.

Fig. 1 illustrates vaporization in and occlusion of an auxiliary artery in the cremaster. Fig. 1A shows the vessel, traced in yellow, prior to the injection of droplet solution, but with ultrasound on as a negative control; Fig. 1B-F show the development of an embolus over the course of 110 seconds. No flow was visible in the upper branch of the artery at 110 seconds post-injection. Some flow was visible in the bottom branch; the distal portion of the embolus had begun to separate from the bulk of the embolus after a minute, as seen in Fig. 1D-F.

These results confirmed the efficacy of embolus formation and the feasibility of using an intravenous injection as a droplet delivery method.

\section{B. Treatment Efficacy}

The changes in tumor volume over a two-week treatment period for a treatment group and for three control groups are plotted in Fig. 2. Ultrasound exposure only, droplet injection only, and saline injection only were used as negative controls. Values are presented as a percentage of the initial tumor volume; mean \pm standard error is plotted for each group $(\mathrm{N}=5)$. Below the graph are three representative B-mode ultrasound images from a treated mouse (Fig. 2A-C). Tumor volume was maintained at approximately baseline in the treatment group, a significant improvement over controls $(\mathrm{p}<0.05)$. Tumor growth was not significantly different between the three control groups.

Similar effects on tumor growth have previously been shown when treating with ADVenhanced clinical high-intensity focused ultrasound ${ }^{[20-21]}$; repeated treatments using ultrasound with microbubble contrast agents have also been reported. ${ }^{[22,23]}$ This work is 
therefore consistent with the existing literature on ADV and cancer therapy, and serves as a novel extension of these previously explored treatment avenues.

\section{Vaporization-Induced Bioeffects}

In the cremaster, both hemorrhage and vasoconstriction were immediately discernable upon the initiation of vaporization. Hemorrhage occurred mainly in smaller capillaries surrounding the larger vessels that were being embolized, while the focal vessel exhibited vasoconstriction. The yellow ellipses in Fig. 1 serve to outline a region of significant hemorrhage and its development over time. The reduction in vessel diameter is most pronounced in the lower branch of the vessel shown in Fig. 1; the approximate vessel boundaries are traced in yellow.

The vasoconstriction was likely due to small scale damage to the artery being embolized, caused specifically by vaporization. While high-amplitude ultrasound is known to induce vasoconstriction or even degeneration of the tunica media on its own, these effects were not observed in this study until the initiation of vaporization. ${ }^{[24-25]}$ Other studies have observed endothelial cell damage in response to ultrasonically-driven microbubble contrast agent and ADV. ${ }^{[26-28]}$ This endothelial damage can lead to vasoconstriction; this process likely explains the observed reduction in vessel diameter. ${ }^{[29]}$

Similar results were observed during the tumor treatments. Significant hemorrhage was visible on the surface of the tumor following treatment, but not with ultrasound alone. The vasoconstriction and hemorrhage are likely beneficial in the context of ischemia induction.

\section{Conclusion}

This study demonstrates the efficacy of using acoustic droplet vaporization for noninvasive arterial embolization. Intravital microscopy experiments illustrated the feasibility of occluding vessels with this method; the mouse experiments expanded upon this result, showing the efficacy of gas embolotherapy as a standalone treatment. Future studies may investigate the concurrent delivery of a chemotherapeutic agent. ADV has previously been shown to increase cell membrane permeability via a process known as sonoporation. ${ }^{[30]}$ The potential for this phenomenon to increase drug uptake in the tumor will be studied, with the goal of subverting the lack of discernable benefit of traditional chemoembolization over bland embolization.

\section{Acknowledgments}

Research supported by the NIH under grant R01EB006476.

\section{References}

[1]. Stuart K "Chemoembolization in the Management of Liver Tumors." The Oncologist, vol. 8, pp. 425-437, 2003. [PubMed: 14530495]

[2]. Blum HE. "Hepatocellular Carcinoma: Therapy and Prevention.” World J Gastroenterol, vol. 11, pp.7391-7400, 2005. [PubMed: 16437707]

[3]. Mojtahedi A, Yang X, Goswami GK. "Embolotherapy in the Management of Hepatocellular Carcinoma.” Semin Intervent Radiol, vol. 25, pp.234-241, 2008. [PubMed: 21326513] 
[4]. Breedis C, Young G. "The Blood Supply of Neoplasms in the Liver." Am J Pathol, vol. 30, pp. 969-985, 1954. [PubMed: 13197542]

[5]. Waghray A, Murali AR, Narayanan Menon KV. "Hepatocellular Carcinoma: From Diagnosis to Treatment.” World J Hepatol, vol. 7, pp.1020-1029, 2015. [PubMed: 26052391]

[6]. Massarweh NN, Davila JA, El-Serag HB, Duan Z, Temple S, May S, Sada YH, Anaya DA. "Transarterial Bland Versus Chemoembolization for Hepatocellular Carcinoma: Rethinking a Gold Standard.” J Surg Res, vol. 200, pp.552-559, 2016. [PubMed: 26507276]

[7]. Tsochatzis EA, Fatourou E, O’Beirne J, Meyer T, Burroughs AK. “Transarterial Chemoembolization and Bland Embolization for Hepatocellular Carcinoma.” World J Gastroenterol, vol. 20, pp.3069-3077, 2014. [PubMed: 24695579]

[8]. Kripfgans OD, Fowlkes JB, Miller DL, Eldevik OP, Carson PL. “Acoustic Droplet Vaporization for Therapeutic and Diagnostic Applications.” Ultrasound Med Biol, vol. 26, pp.1177-1189, 2000. [PubMed: 11053753]

[9]. Fabiilli ML, Lee JA, Kripfgans OD, Carson PL, Fowlkes JB. "Delivery of water-soluble drugs using acoustically triggered perfluorocarbon double emulsions.” Pharm Res, vol. 27, pp.27532765, 2010. [PubMed: 20872050]

[10]. Rapoport NY, Kennedy AM, Shea JE, Scaife CL, Nam KH. "Controlled and Targeted Tumor Chemotherapy by Ultrasound-activated Nanoemulsions/Microbubbles." J Control Release, vol. 138, pp.268-276, 2009. [PubMed: 19477208]

[11]. Juliar BA, Bromley MM, Moncion A, Jones DC, O’Neill EG, Wilson CG, Franceschi RT, Fabiilli ML. "In Situ Transfection by Controlled Release of Lipoplexes Using Acoustic Droplet Vaporization.” Adv Healthc Mater, vol. 5, pp.1764-1774, 2016. [PubMed: 27191532]

[12]. Qamar A, Wong ZZ, Fowlkes JB, Bull JL. "Dynamics of Acoustic Droplet Vaporization in Gas Embolotherapy.” Appl Phys Lett, vol. 96, pp.143702, 2010. [PubMed: 20448802]

[13]. Sheeran PS, Rojas JD, Puett C, Hjelmquist J, Arena CB, Dayton PA. "Contrast-Enhanced Ultrasound Imaging and in Vivo Circulatory Kinetics with Low-Boiling-Point Nanoscale PhaseChange Perfluorocarbon Agents.” Ultrasound Med Biol, vol. 41, pp.814-831, 2015. [PubMed: 25619781]

[14]. Kripfgans OD, Fowlkes JB, Woydt M, Eldevik OP, Carson PL, "In vivo droplet vaporization for occlusion therapy and phase aberration correction," IEEE Trans Ultrason Ferroelect Freq Control, vol. 49, pp.726-738, 2002.

[15]. Samuel S, Duprey A, Fabiilli ML, Bull JL, Fowlkes JB. "In Vivo Microscopy of Targeted Vessel Occlusion Employing Acoustic Droplet Vaporization.” Microcirculation, vol. 19, pp.501-509, 2012. [PubMed: 22404846]

[16]. Zhang M, Fabiilli ML, Haworth KJ, Fowlkes JB, Kripfgans OD, Roberts WW, Ives KA, Carson PL. "Initial Investigation of Acoustic Droplet Vaporization for Occlusion in Canine Kidney." Ultrasound Med Biol, vol. 36, pp.1691-1703, 2010. [PubMed: 20800939]

[17]. Parsons JE, Cain CA, Fowlkes JB. "Cost-effective assembly of a basic fiber-optic hydrophone for measurement of high-amplitude therapeutic ultrasound fields.” J Acoust Soc Am, vol. 119, pp. 1432-40, 2006. [PubMed: 16583887]

[18]. Tomayko MM, Reynolds CP. "Determination of subcutaneous tumor size in athymic (nude) mice." Cancer Chemother Pharmacol, vol. 24, pp.148-154, 1989. [PubMed: 2544306]

[19]. R Core Team. R: A language and environment for statistical computing. Vienna, Austria: R Foundation for Statistical Computing, 2016.

[20]. Zhu M, Jiang L, Fabiilli ML, Xu LX, Zhang A, Fowlkes JB. "Treatment of murine tumors using acoustic droplet vaporization enhanced high intensity focused ultrasound.” Phys Med Biol vol. 58, pp.6179-91, 2013. [PubMed: 23948709]

[21]. Ho YJ, Yeh CK. "Concurrent anti-vascular therapy and chemotherapy in solid tumors using drugloaded acoustic nanodroplet vaporization.” Acta Biomater, vol. 49, pp.472-485, 2017. [PubMed: 27836803]

[22]. Chien Ting C, Raju BI, Shevchenko T Klibanov AL. "Control and reversal of tumor growth by ultrasound activated microbubbles." in 2009 IEEE International Ultrasonics Symposium, pp.77$80,2009$. 
[23]. Wood AKW, Ansaloni S, Ziemer LS, Lee WMF, Feldman MD, Sehgal CM. "The antivascular action of physiotherapy ultrasound on murine tumors." Ultrasound Med Biol, vol. 31, pp.14031410, 2005. [PubMed: 16223644]

[24]. Ishikawa T, Okai T, Sasaki K, Umemura S, Fujiwara R, Kushima M, Ichihara M, Ichizuka K. "Functional and histological changes in rat femoral arteries by HIFU exposure." Ultrasound Med Biol, vol. 29, pp.1471-7, 2003. [PubMed: 14597344]

[25]. Hynynen K, Chung AH, Colucci V, Jolesz FA. "Potential adverse effects of high-intensity focused ultrasound exposure on blood vessels in vivo." Ultrasound Med Biol, vol. 22, pp.193201, 1996. [PubMed: 8735529]

[26]. Hwang JH, Brayman AA, Reidy MA, Matula TJ, Kimmey MB, Crum LA. "Vascular effects induced by combined 1-MHz ultrasound and microbubble contrast agent treatments in vivo." Ultrasound Med Biol, vol. 31, pp.553-564, 2005. [PubMed: 15831334]

[27]. Hwang JH, Tu J, Brayman AA, Matula TJ, Crum LA. "Correlation Between Inertial Cavitation Dose and Endothelial Cell Damage In Vivo.” Ultrasound Med Biol, vol. 32, pp.1611-1619, 2006. [PubMed: 17045882]

[28]. Seda R, Li D, Fowlkes JB, Bull JL. "Characterization of Bioeffects on Endothelial Cells under Acoustic Droplet Vaporization.” Ultrasound Med Biol, vol. 41, pp.3241-3252, 2015. [PubMed: 26403698]

[29]. Loscalzo J "Endothelial Injury, Vasoconstriction, and its Prevention.” Tex Heart Inst J, vol. 22, pp.180-184, 1995. [PubMed: 7647603]

[30]. Fix SM, Novell A, Yun Y, Dayton PA, Arena CB. "An evaluation of the sonoporation potential of low-boiling point phase-change ultrasound contrast agents in vitro.” J Ther Ultrasound, vol. 5, pp.7-17, 2017. [PubMed: 28127427] 


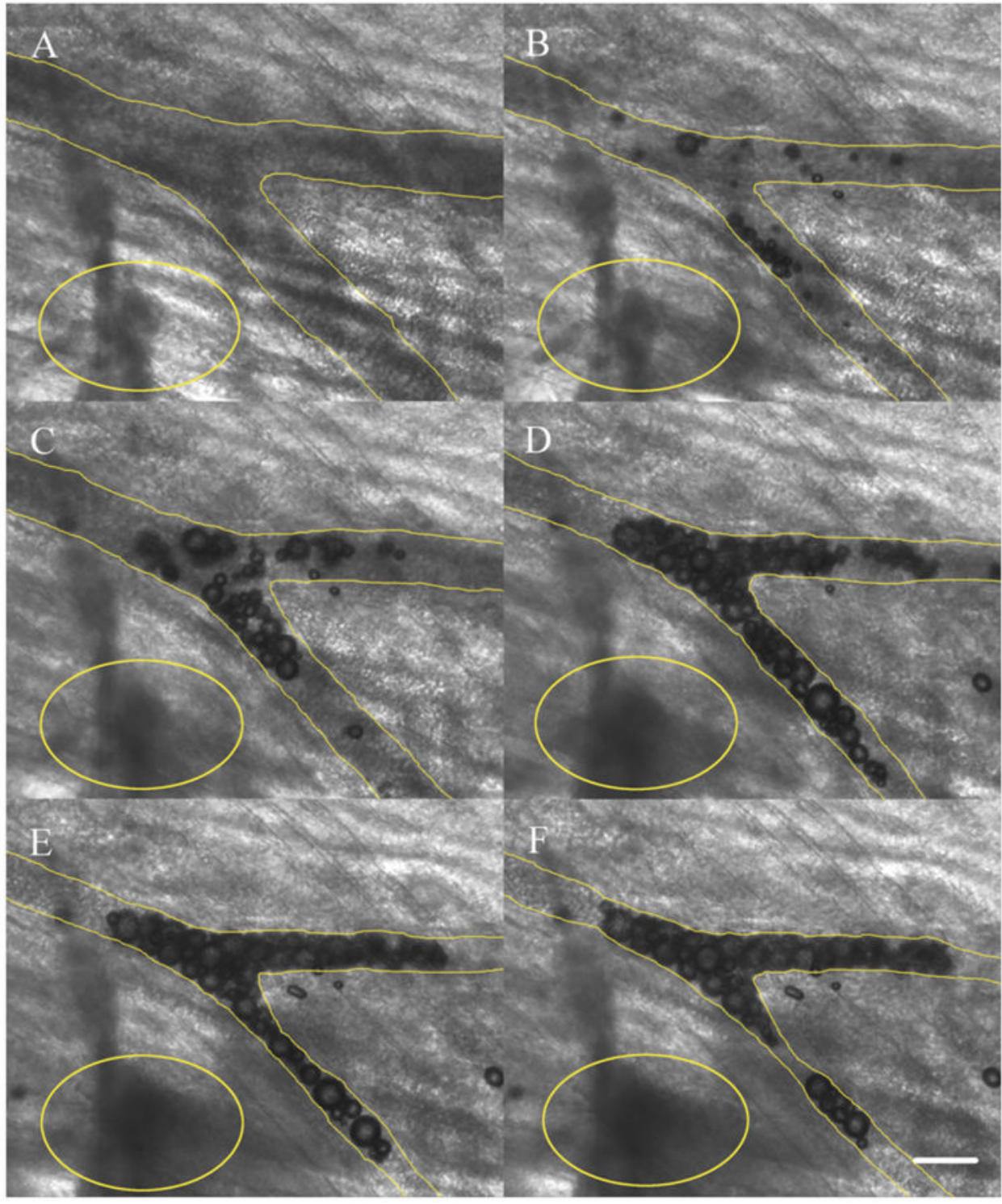

Figure 1.

Vaporization and occlusion in an auxiliary artery in the rat cremaster. A) Before injection of droplet solution. B-F) 20, 30, 60, 85, and 110 seconds post-injection respectively. The tissue was exposed to ultrasound during the entire time course. The yellow ellipses highlight the hemorraghic region. Approximate vessel boundaries are traced in yellow. Scale bar $=100$ $\mu \mathrm{m}$. 

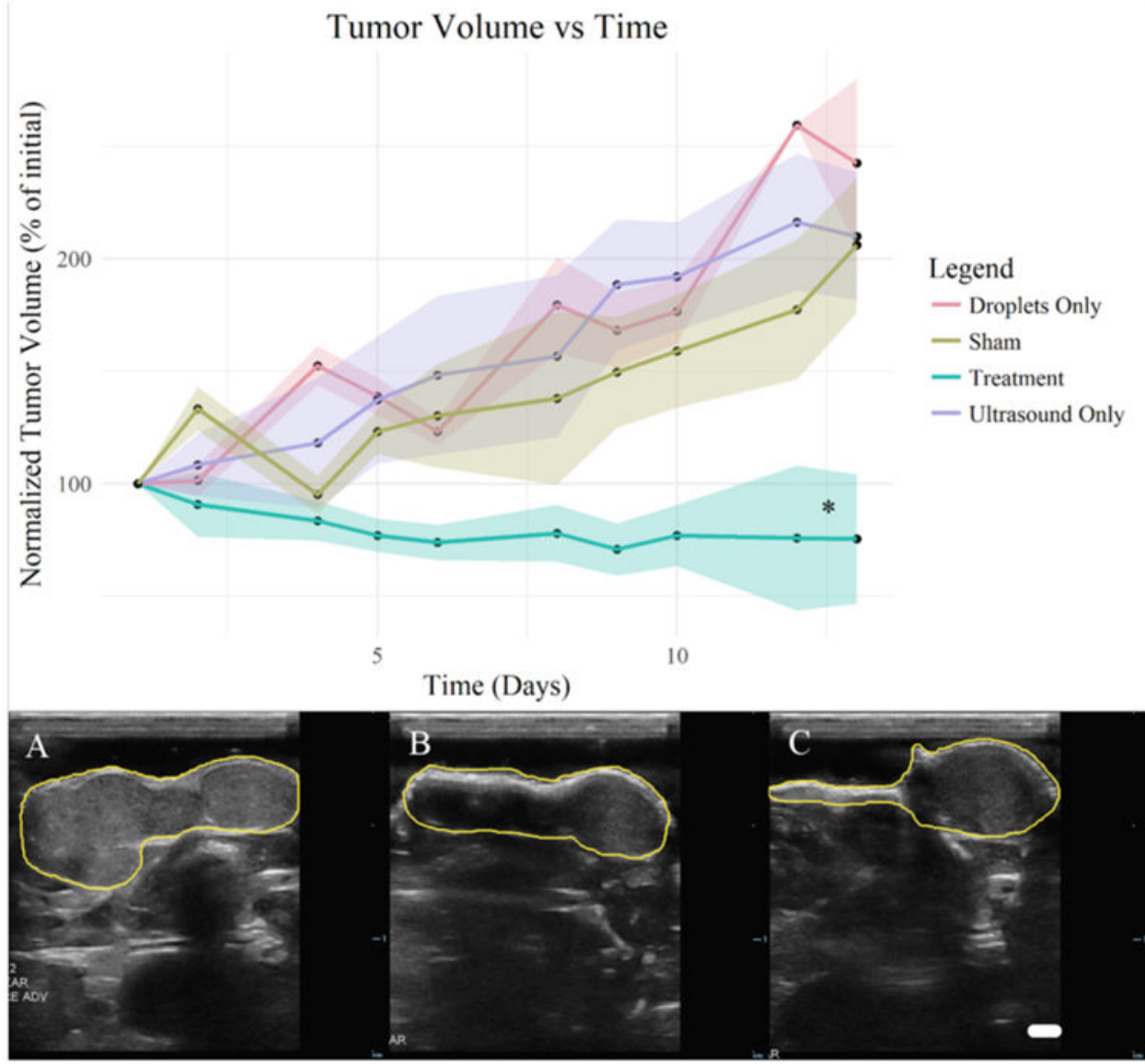

Figure 2.

Normalized tumor volume over a two-week treatment course. A-C) B-mode ultrasound images of the same treated tumor at Day 1, 6, and 14 respectively. Approximate tumor boundaries are shown in yellow. Scale bar $=1 \mathrm{~mm} .\left(^{*}=p<0.05\right.$. $)$ 